\title{
Lumen
}

Selected Proceedings from the Canadian Society for Eighteenth-Century Studies

\section{Du secret d'État au for intérieur : tourments du vieil âge dans l'Entretien avec moi même de Jacques Necker}

\section{Catherine Dubeau}

Volume 37, 2018

URI : https://id.erudit.org/iderudit/1042224ar

DOI : https://doi.org/10.7202/1042224ar

Aller au sommaire du numéro

Éditeur(s)

Canadian Society for Eighteenth-Century Studies / Société canadienne d'étude du dix-huitième siècle

ISSN

1209-3696 (imprimé)

1927-8284 (numérique)

Découvrir la revue

Citer cet article

Dubeau, C. (2018). Du secret d’État au for intérieur : tourments du vieil âge dans l'Entretien avec moi même de Jacques Necker. Lumen, 37, 75-89.

https://doi.org/10.7202/1042224ar d'utilisation que vous pouvez consulter en ligne. 


\title{
Du secret d'État au for intérieur: tourments du vieil âge dans l'Entretien avec moi même de Jacques Necker
}

\author{
Catherine Dubeau
}

University of Waterloo

Je puis m[']exprimer ainsi en ne parlant qu'a moi'.

Jacques Necker

Parmi les manuscrits légués par Jacques Necker, il en est peu d'aussi intimes que l'Entretien avec moi même, rédigé à l'époque du Consulat. Conservés dans les archives du château de Coppet, en Suisse, le brouillon (intitulé simplement Entretien avec moi meme) et la version remaniée (Entretien avec moi même: Motifs pour ou contre mon retour en France $^{2}$ ) n'ont jusqu'ici fait l'objet d'aucune édition intégrale. Les deux textes ne sont pas des autographes mais consistent en copies plus ou moins annotées de la main de Necker. À notre connaissance, seuls quelques fragments ont été publiés par Auguste de Staël (CEuvres complètes de M. Necker, 1820-182133), le comte d'Haussonville (Madame de Staël et M. Necker, 1925'), Édouard Chapuisat (Necker (1732-1804),

1. Jacques Necker, «Entretien avec moi meme », archives de Coppet, 16 feuillets, f. $2 r^{\circ}$. Dorénavant, nous utiliserons le sigle EM I. Nos remerciements et notre reconnaissance la plus profonde vont à feu Monsieur le comte d'Haussonville, grâce à qui nous avons pu consulter ces archives.

2. Archives de Coppet, 17 feuillets. Dorénavant, nous utiliserons le sigle EM II.

3. Auguste de Staël, «Notice sur M. Necker, par A. de Staël Holstein, son petitfils », Euvres complètes de M. Necker, publiées par M. le baron de Staël, son petit-fils, Paris, Treuttel et Würtz, 1820-1821, t. I, p. CCCXLVII.

4. Gabriel-Paul-Othenin d'Haussonville, Madame de Staël et M. Necker d'après leur correspondance inédite, Paris, Calmann-Lévy, 1925, p. 197-202. 
193855), Béatrix d'Andlau (La jeunesse de Madame de Staël : 1766-1786, $1970^{6}$ ) et Ghislain de Diesbach (Necker ou la faillite de la vertu, 19787). Là où le lecteur s'attend à une réflexion de nature politique, se déploie la voix d'un homme fragilisé par son grand âge et tourmenté par les instances répétées de sa fille, désireuse de le voir quitter Coppet pour Paris. Necker soupèse le pour et le contre d'un tel déplacement. Dans la foulée, passé et présent, jeunesse et vieillesse, carrière politique, vie mondaine et retraite solitaire passent au crible de la comparaison. Éditeur des Euvres complètes de son grand-père, Auguste de Staël soulignait dès 1821 la nature privée de l'exercice en évoquant ces «notes [de Necker] destinées pour lui seul ${ }^{8} »$. Troublantes d'authenticité, ces pages lèvent le voile sur un homme méconnu et dont seule Germaine de Staël et son fils Auguste avaient pu donner quelques aperçus dans leur préface respective9. L'Entretien avec moi même nous permet d'aller plus avant dans notre connaissance de Necker en montrant à l'œuvre une écriture introspective manifestement chère à l'auteur, à un moment où il se trouve en proie au doute et au déchirement intérieur. Le présent article souhaiterait amorcer l'analyse des manuscrits en portant son attention sur trois éléments fondamentaux du processus d'autoreprésentation, soit le triple rapport de Necker à sa fille (Madame de Staël), à sa condition d'homme vieillissant, enfin à son passé de ministre. En abordant de front le problème du lieu de vie, Necker pose une question de nature particulière tout autant que générale: quelle place pour le vieillard dans cette France consulaire, écartelée entre Révolution et Empire?

Les manuscrits de l'Entretien ne peuvent être datés avec précision, cependant quelques indices nous permettent d'établir les limites temporelles de leur rédaction. Necker répète à plusieurs reprises qu'il écrit alors qu'il a quitté la France depuis 10 ans - une occurrence indique

5. Édouard Chapuisat, Necker (1732-1804), Paris, Librairie du recueil Sirey, 1938, p. $257-260$.

6. Béatrix d'Andlau, La jeunesse de Madame de Staël: 1766-1786, Genève, Droz, 1970, p. 53, 113-114.

7. Ghislain de Diesbach, Necker ou la faillite de la vertu, Paris, Perrin, 2004 [1978], p. $43^{8}$.

8. Auguste de Staël, op. cit., p. CCCXLVIJ.

9. Ibid., p. I-CCCLJ et Germaine de Staël, «Du caractére de M. ${ }^{r}$ Necker et de sa vie privée ", Manuscrits de M.r Necker, publiés par sa fille, Genève, Paschoud, 1804, p. 1-153. 
«dix ou douze ans ${ }^{10}$ ». Or le départ de France a lieu en septembre 1790, dans les circonstances très difficiles que l'on sait. En effet, le troisième ministère de Necker s'achève sur une démission le 3 septembre 1790, alors que l'homme d'État ne jouit plus de la confiance royale et que l'Assemblée n'est plus favorable à ses conseils. Le point de rupture est atteint lorsqu'il se trouve personnellement menacé - tout comme les autres ministres - par une foule cherchant à se venger de l'écrasement violent d'une mutinerie de soldats ayant eu lieu quelques jours auparavant à Nancy ${ }^{11}$. Lorsque La Fayette lui suggère de s'abriter en lieu sûr, Necker écrit à l'Assemblée pour annoncer sa démission et se réfugie définitivement en Suisse, au château de Coppet acquis en 1784 . Ainsi, la chronologie nous permet d'émettre l'hypothèse selon laquelle les textes auraient été rédigés vers 1800 , ce qui correspond également à l'opinion de Béatrix d'Andlau ${ }^{12}$. Par ailleurs, trois allusions supplémentaires nous guident: d'une part, le texte n'a pas pu être rédigé avant le 20 mai 1800 puisque cette date correspond à la rencontre de Necker et Bonaparte à Genève, dont un passage de l'Entretien fait brièvement mention: «Il [le premier Consul] ne m'invita point de venir a Paris lorsqu'il me vit a Geneve ${ }^{13}$.» D'autre part, dans son manuscrit, Necker anticipe avec crainte la réception du «roman» de Madame de Staël. Il s'agit bien entendu de Delphine, qui paraîtra au mois de décembre 1802: "Voila ma fille aussi qui avec son roman va se faire des ennemis parmi les Catholiques zelés, et même parmi les nombreux antagonistes des idées philosophiques ${ }^{14}$. Enfin, il est fait mention de la menace

10. EM II, f. $8 \mathrm{r}^{\circ}$.

11. Voir Léonard Burnand, Les pamphlets contre Necker. Médias et imaginaire politique au XVIII ${ }^{e}$ siècle, Paris, Classiques Garnier, 2009, p. 295.

12. Voir Béatrix d'Andlau, op. cit., p. 53.

13. EM I, f. $5 \mathrm{v}^{\circ}$. Madame de Staël relatera les circonstances de cette rencontre (Considérations sur la Révolution française, Paris, Tallandier, 2000 [1983], p. 377): «M. Necker eut un entretien avec Bonaparte à son passage en Italie par le mont Saint-Bernard, peu de temps avant la bataille de Marengo; pendant cette conversation, qui dura deux heures, le premier consul fit à mon père une impression assez agréable, par la sorte de confiance avec laquelle il lui parla de ses projets futurs. » Madame de Staël évoque par la suite la colère de Bonaparte à la lecture des Dernières vues de politique et de finances (1802) de Necker, qui vantaient les mérites du premier Consul tout en se permettant de commenter ses projets: «Aussi, dès que cet ouvrage parut, les journalistes reçurent-ils l'ordre de l'attaquer avec le plus grand acharnement. Bonaparte signala M. Necker comme le principal auteur de la révolution. »

14. EM II, f. gr $^{\circ}$. 
d'un «éclat scandaleux par les affaires de $\mathrm{M}^{\mathrm{r}}$ de St. ${ }^{15}$ » (Éric de Staël, époux de Madame de Staël) qui sont au plus mal depuis déjà longtemps. Or, le décès de M. de Staël surviendra le 9 mai 1802. À la lumière de ces indices, nous pouvons affirmer que les textes ont été rédigés entre le 20 mai 1800 et le 9 mai 1802, et vraisemblablement plus tard que tôt puisque Delphine devait être bien entamé sinon achevé pour susciter les commentaires précédents.

Notons ici quelques différences significatives entre le brouillon et la seconde version de l'Entretien: de façon générale, le premier texte adopte un ton plus pessimiste que le second, les arguments en faveur du déplacement en France occupent moins de deux feuillets et sont expédiés au profit d'un long développement en défaveur du déménagement. Les deux volets du texte sont soulignés de telle sorte qu'ils laissent entrevoir l'exigence de vérité et le ton confessionnel rattachés à l'examen de conscience auquel se prête Necker: "Je ne crois pas avoir dissimulé quoi qu’en[?] < dans cet> abregé les motifs qui m’appelleroient à Paris. Je vais rendre compte avec la même vérité de la réunion de circonstances qui combattent ce plan ${ }^{16}$. » À certains égards, le brouillon apparaît plus spontané et plus immédiatement évocateur du tourment et de l'inquiétude qui habitent l'auteur au moment de la rédaction. Nous verrons par les citations proposées plus bas que cette version initiale formule de façon très directe les opinions défavorables de Necker quant aux obligations de la vie mondaine, et qu'il exprime sans détour les détails les plus embarrassants associés à sa condition d'homme vieillissant. Quant à elle, la seconde version de l'Entretien semble avoir fait l'objet d'un remaniement visant à en policer l'expression et à rééquilibrer l'espace accordé aux arguments dans le cadre de deux parties respectivement intitulées «Pour» et «Contre mon changement de Domicile». Le ton de cette seconde version apparaît davantage tempéré et certains passages font l'objet de modifications sensibles: celui concernant le tombeau de son épouse est considérablement augmenté, tandis que les soucis financiers de M. de Staël sont passés sous silence. L'édition critique que nous nous proposons d'entreprendre $^{17}$ offrira un compte rendu exhaustif de ces différences. Dans

15. EM I, f. $15 \mathrm{v}^{\circ}$.

16. Ibid., f. $2 \mathrm{r}^{\circ}$.

17. À paraître dans les Cahiers staëliens. 
le cadre du présent article, nous référerons alternativement aux deux versions.

Le brouillon de l'Entretien s'ouvre sur une demande de Madame de Staël qui «desire avec ardeur avec passion ${ }^{18}$ » voir son père s'établir à Paris. À l'époque où ce vœu est formulé, la romancière fait régulièrement l'aller-retour entre la France et la Suisse afin de préserver sa vie mondaine de même que ses liens avec un père profondément aimé, résidant tantôt à Coppet, tantôt à Genève. Mais ces voyages, explique Necker, l'épuisent, sans compter qu'ils compliquent singulièrement tout plan d'éducation suivi pour ses deux garçons, Auguste (1790-1827) et Albert (1792-1813) : «Ma fille encore est peinée avec raison du defaut d'unité dans ses plans d'education pour ses enfans, effet inevitable de ses deplacemens annuels ${ }^{19}$. » La sécurité de leur jeune sœur, Albertine (1797-1838), est également compromise par cette mobilité : «C'est aussi un risque que ces voyages continuels, et il s'accroit en imagination, maintenant qu'une petite fille devrait être menée et ramenée tous les $a n s^{20}$.» L'installation à Paris permettrait ainsi à Necker d'assurer une plus grande stabilité à toute la famille et de contrer la «solitude de Coppet», sans compter que le «[c]limat doux» de la France, la «varieté» des produits de table de même que le «mouvement d'une grande $v$ ille ${ }^{21} »$ seraient susceptibles de lui plaire. L'idée d'attirer son père à Paris procède aussi, pour Madame de Staël, de la conviction qu'il jouera pour elle le rôle d'un protecteur et, par sa considération, constituera une « Egide $^{22}$ », « une sauvegarde ${ }^{23}$ » qui la mettra hors d'atteinte d'un gouvernement déjà fort hostile à son endroit. Necker écrit qu'elle «n'est pas en faveur auprès du Consul ${ }^{24}$ » et, dans le brouillon, qu'elle «est mal avec la pluspart des personnes en puissance et en credit a Paris $^{25} »$. On sait que dès octobre 1803 , le séjour à Paris sera interdit à Madame de Staël, ce qui l'incitera à partir pour l'Allemagne. Elle en tirera les célèbres pages de l'essai De l'Allemagne qui lui vaudra la censure en 1810, et l'assignation à résidence à Coppet et Genève.
18. EM I, f. $1 r^{\circ}$.
19. EM II, f. $4 \mathrm{r}^{\circ}$.
2o. Ibid., f. $6 \mathrm{r}^{\circ}$.
21. Ibid., f. $6 \mathrm{v}^{\circ}-7 \mathrm{r}^{\circ}$.
22. EM I, f. $6 v^{\circ}$.
23. EM II, f. $3 \mathrm{v}^{\circ}$.
24. Ibid., f. $2 \mathrm{v}^{\circ}$.
25. EM I, f. $1 v^{\circ}$. 
Bientôt, elle fuira à l'avancée des troupes napoléoniennes et se déplacera vers l'Angleterre en passant par l'Autriche, la Russie et la Suède, avant de revenir à Paris après l'abdication de Napoléon. Le souvenir de ce périple sera restitué dans Dix années d'exil (1821). Ainsi, dès 18001802, Necker se fait prudent: «Tout tremble, tout flechit devant le Dieu du jour.... [sic] serai-je même une sauvegarde pour ma fille ${ }^{26}$ ? » Bien qu'il se montre très sensible au sort de Madame de Staël, et désireux de lui porter secours, Necker ne semble s'autoriser aucune décision sans avoir préalablement évalué et soupesé, par écrit, les avantages et désavantages d'un retour dans sa patrie d'adoption.

Le premier argument en faveur du déménagement est tout naturellement lié au désir de ne plus quitter sa fille, à laquelle il voue une affection fervente. Sous la plume de son père, Germaine de Staël apparaît d'abord comme une femme soucieuse d'entretenir sa vie sociale à Paris. Ce rapport passionné à la vie mondaine, dont toute l'œuvre et la correspondance staëliennes témoignent par ailleurs, ouvre la première section du manuscrit:

Il n'y a que ce moïen d'eviter une separation longue et frequente entre ma fille et moi; car rien n'est plus démontré, rien n'est plus invincible que son gout pour vivre a Paris. Les habitudes de son education, de son enfance, de sa premiere jeunesse lui ont inspiré ce gout; et son besoin de mouvement, le mal physique que lui ferait l'ennui de la solitude, et même la monotonie de Genêve, ont converti par degrés son eloignement pour ce Pays dans une sorte d'antipathie portée maintenant a l'extrême ${ }^{27}$.

Rappelons que Germaine de Staël a été dès le plus jeune âge intégrée au salon tenue par sa mère à Paris. De spectatrice discrète, elle est rapidement devenue une participante remarquable et très sollicitée par les visiteurs éblouis devant la vivacité de son esprit et l'intelligence d'une conversation désormais passée à l'histoire ${ }^{28}$. À ce sujet, Necker ne tarit pas d'éloges et enchaîne ici sur un bref panégyrique dont on trouve d'autres exemples dans son œuvre:

26. EM II, f. $16 v^{\circ}$.

27. Ibid., f. $1 \mathrm{r}^{\circ}$.

28. Voir à ce sujet le témoignage de Catherine Rilliet Huber, «Notes sur l'enfance de Madame de Staël », Cahiers staëliens, 60, 2009, p. 61-73. 
Il est tems de dire que ce n'est pas seulement le besoin absolu de distractions qui rend la vie de Paris si necessaire a ma fille, c'est encore le plaisir naturel attaché au talent surprenant de conversation que la nature lui a donné. Ce talent est sans bornes qu[?] <comme> sans modele; richesse et varieté d'idées; graces dans les formes et aptitude singuliere a traiter tous les sujets, enfin une facilité d'elocution qui ressemble a la marche et a la velocité du vent. Où trouver le champ propre a l'exercice d'un pareil talent? Le theatre de Paris, ce theatre unique en son genre peut a peine y suffire. Rien n'est donc plus naturel que le gout passionné de ma fille pour Paris $[\ldots]^{29}$.

Si le portrait de Madame de Staël est généralement élogieux dans le cadre des manuscrits, Necker est aussi très franc sur l'existence de fortes tensions qui minent sa relation avec sa fille. Il exprime ses craintes au sujet de la «brouillerie ouverte ${ }^{30}$ » qui ne manquera pas de survenir s'il n'accède pas à ses désirs, et souligne son impulsivité : «Ma fille n'est pas maitre de son caractere ${ }^{31}$ !» Il décrit l'impression que produit sur lui son mécontentement et les disputes qui s'ensuivent: «Ce spectacle est insupportable pour moi, et d'autant plus que la situation penible de ma fille amêne continuellement des plaintes des reproches de sa part, et souvent des controverses douloureuses auxquelles mes forces ne sont pas toujours proportionnées ${ }^{32}$.» En outre, il ne saurait passer sous silence les erreurs, sinon les dangers auxquels Madame de Staël s'expose en raison de sa vivacité - en particulier dans ses ouvrages et au sein des conversations:

Ma fille dit que ma presence a Paris la rendrait plus prudente, et que la crainte peut être de deranger une derniere fois ma situation nouvelle guiderait ses demarches et ses discours. C'est un problème, car d'un autre coté croyant sa fixation en France plus assurée par ma presence, elle aurait des motifs pour se hazarder autant et plus en conversation. Je crois bien cependant que pour toutes sortes de resolutions reflechies, pour tout ce qui ne tiendrait pas a la vivacité de la parole, la proximité de mes conseils l'inviterait a en jouïr et je ne doutes [sic] point pour ces cas là de sa deference ${ }^{33}$.

29. EM II, f. $1 \mathrm{v}^{\circ}-2 \mathrm{r}^{\circ}$.

30. Ibid., f. $7 \mathrm{r}^{\circ}$.

31. Ibid., f. $17 \mathrm{r}^{\circ}$.

32. Ibid., f. $1 \mathrm{v}^{\circ}-2 \mathrm{r}^{\circ}$.

33. Ibid., f. $5 \mathrm{r}^{\circ}-5 \mathrm{v}^{\circ}$. 
Quoique la perspective de profiter en tout temps de sa famille soit, dans le brouillon comme dans la seconde version, le plus grand argument en faveur de son retour en France, Necker n’hésite pas à tempérer l'enthousiasme de Madame de Staël en regard de l'aide ou du bonheur qu'il pourrait lui apporter, ainsi qu'à ses enfants ${ }^{34}$. C'est qu'elle ne semble pas tenir compte d'un facteur d'importance: l'âge de son père qui, en 1800, approche les soixante-dix ans (Necker est né en 1732): «[...] je suis un debile roseau pour la servir surtout de près et en exposant ce qui me reste un [sic] peu de consideration ${ }^{35}$.» S'amorce alors un «retour triste ${ }^{36}$ » sur soi, une pénible représentation de sa personne comme vieillard qui irrigue l'ensemble des manuscrits et en trahit la nature profondément intime. En guise d'exemple, nous citerons quelques extraits particulièrement émouvants:

Je continue l'exposition de mes reflexions. Je me fais beaucoup de peine de me montrer en France après une si longue absence, de m'y montrer dans un âge si près de la decrepitude, au lieu de rester dans cette ombre où l'on vieillit silencieusement. Je me fais beaucoup de peine de reparaitre en France si près du moment où l'on a besoin d'indulgence et de pitié. Je ne puis souffrir non plus avec l'idée d'un deplacement cette corpulence qui m’a rendu un etre si visible et si remarquable, et ces jambes grossies qui rendent presque ridicule aux yeux des autres, de nouveaux plans et une nouvelle carriere. Ma fille me demande pour la soutenir a Paris, et ceux qui me verront passer croiront quelle me mêne a des eaux ${ }^{37}$.

Le brouillon du manuscrit aborde avec encore plus de franchise l'ampleur de la «detresse» qui habite Necker alors que les années passent et que les infirmités gagnent son corps, comme en témoigne d'ailleurs une chaise roulante de bois encore visible au musée du château de Coppet. En effet, après avoir exprimé l'embarras extrême

34. Voir Ibid., f. $4 \mathrm{r}^{\circ}-4 \mathrm{v}^{\circ}$ : «Ma fille en s'occupant de sa famille, croit que ma presence a Paris favoriserait essentiellement le sort futur de ses enfans, et c'est une opinion que je ne puis partager. Je serai tout au moins vieux et très vieux selon les lois de la nature, lorsqu'il sera question de fixer leur etat dans le monde, et ce n'est jamais dans ce $[$ sic $]$ periode de la vie qu'on a du credit. Si donc je ne puis esperer de les servir que par mon ombre, il faut la tenir belle autant que possible, et pour atteindre a ce but, je crois que la retraite vaut mieux que mon retour au milieu du monde. »

35. Ibid., f. $7 \mathrm{v}^{\circ}$.

36. Id.

37. Ibid., f. $9 \mathrm{r}^{\circ}-9 \mathrm{v}^{\circ}$. 
que lui causerait l'obligation de «faire les honneurs d'une maison » et d'accueillir des « compagnies desassorties » - il emploie explicitement le mot de "galere ${ }^{38}$ » pour désigner la reprise éventuelle d'une vie sociale à son château de Saint-Ouen, situé en banlieue de Paris Necker revient avec émotion sur sa condition physique actuelle:

Ma santé viendroit encore rendre penible le nouveau trin $<$ train $>$ de vie auquel je serois appellé est $<$ et $>$ c'est ici que je ne puis dissimuler que l'etat particulier de mes dents me met souvent dans l'embarras et surtout a table car je ne puis plus parler en mangeant ou il faut que je me donne le tems de placer les aliments d'une certaine maniere pour entamer un propos et pour être en état de repondre a une interrogation. Tout cela est misérable à dire miserable a avouer mais c'est ma detresse actuelle et je n'ai a prevoir que le pis et non aucune amelioration. Enfin par des considerations de tout genre je me sentirois humilié a tous les instants du jour et sil m'arrivoit de l'être a mes propres yeux je ne serois plus rien du tout car ce qu'il y a de bien dans ma nature morale ne peut se passer d'un sentiment de dignité il est en moi une condition essentielle de mon esprit et de mon caractere de mes manieres et sans lui je serois un être eperdu ${ }^{39}$.

Ce passage nous ramène de manière poignante à la nature non seulement spirituelle, mais aussi matérielle et physique des échanges mondains, indissociables d'une maîtrise et d'une représentation de soi reposant en grande partie sur le $\operatorname{corps}^{40}$. À plusieurs reprises, qu'il s'agisse de prendre acte de son poids, de l'état de ses dents, de sa che-

38. EM I, f. $9 \mathrm{r}^{\circ}-9 \mathrm{v}^{\circ}$. Un autre passage du brouillon traduit avec éloquence le rapport difficile de Necker à la sociabilité (ibid., f. $8 \mathrm{v}^{\circ}$ ): «Je ne suis pas un homme aimable comme Volt... Ou comme $\mathrm{M}^{\mathrm{dme}}$ de $\mathrm{S}$. et la circonspection absolument necessaire dans ma position moteroit encore tous les sujets où le caractere peut donner du mouvement a lesprit. Resteroit les conversations sur la litterature je n'y aurois aucun avantage et je laisserois parler les autres sans cesse. »

39. Ibid., f. $9 \mathrm{r}^{\circ}-10 \mathrm{r}^{\circ}$.

40. Les Mélanges (1798) et Nouveaux mélanges (1801) de Madame Necker contiennent à cet égard de nombreuses prescriptions, comme nous le soulignons dans «L'épreuve du salon ou le monde comme performance », La lettre et la mère. Roman familial et écriture de la passion chez Suzanne Necker et Germaine de Staël, Québec/ Paris, PUL/Hermann, 2013, p. 89-117. Voir également Philippe Raynaud, La politesse des Lumières. Les lois, les moeurs, les manières, Paris, Gallimard, 2013; Antoine Lilti, Le monde des salons. Sociabilité et mondanité à Paris au XVIII siècle, Paris, Fayard, 2005; Georges Vigarello (édit.), Histoire du corps, Paris, Seuil, 2005, t. I (De la Renaissance aux Lumières); Alain Montandon (édit.), Dictionnaire raisonné de la politesse et du savoir-vivre. Du Moyen Âge à nos jours, Paris, Seuil, 1995. 
velure blanchie, enfin de son allure générale, Necker apparaît honteux, par avance diminué par les humiliations dont il anticipe faire l'objet, soulignant au passage l'impossibilité pour lui de négocier en personne la récupération du dépôt d'argent fait au Trésor royal plusieurs années avant la Révolution ${ }^{41}$. Madame de Staël rapporte dans la préface aux manuscrits de son père ce rapport douloureux au vieillissement:

Il lui étoit pénible d'être vieux; sa taille qui étoit devenue très grosse, et qui lui rendoit les mouvemens difficiles, lui causoit un sentiment de timidité qui le détournoit d'aller dans le monde. Il ne montoit presque jamais en voiture quand on le regardoit: il ne se promenoit pas quand il pouvoit être vu: Enfin, son imagination aimoit la grâce et la jeunesse, et il me disoit quelquefois: - je ne sais pourquoi je suis humilié des infirmités de l'âge, mais enfin je sens que je le suis ${ }^{42}$.

Necker répète à plus d'une reprise le risque énorme que représenterait pour lui le fait de «reparoitre tout a coup sur le Theatre du monde ${ }^{43}$ ». Lopposition entre le monde et la retraite constitue ainsi un fil conducteur essentiel dans les deux versions de l'Entretien. On se souviendra des nombreuses pensées de son épouse à cet égard: «Toutes les relations avec le grand monde sont au désavantage des vieillards; ainsi ils doivent regarder comme gagné en bonheur et en considération, le tems qu'ils peuvent dérober à la société; car ils n'ont plus qu'à perdre dans le monde ${ }^{44}$.» La conclusion du second manuscrit y fait écho : «Plus on connait les hommes, plus on doit chercher a vivre dans la solitude, lorsque l'âge avancé ne permet plus de de les servir ${ }^{45}$. " L'ère consulaire, préparant le terrain à l'Empire napoléonien, contribue à repenser la valeur individuelle à l'aune d'un idéal militaire valorisant la bravoure et conférant au corps jeune, agile et puissant une place de choix, reléguant de facto l'homme vieillissant - généralement inapte à l'ac-

41. EM II, f. $5 \mathrm{r}^{\circ}:$ : [...] mon âge, mon ancienne situation sont un obstacle absolu a des negotiations de ce genre. Je ne ferais que me rabaisser et l'on me negligerait avec plus d'aisance. Il faut que je fasse ma demande par lettre et de Coppet, il faut que je la fasse d'un lieu où tout ce qui me reste (une vielle [sic] consideration) est encore dans sa valeur, il faut aussi que je tâche de ne pas eveiller les ennemis par ma presence $[\ldots]$..»

42. Germaine de Staël, «Du caractère de M. ${ }^{\mathrm{r}}$ Necker [...] », op. cit., p. 121-122.

43. EM I, f. $3 \mathrm{r}^{\circ}-3 \mathrm{v}^{\circ}$.

44. Suzanne Necker, Mélanges extraits des manuscrits de Madame Necker, Paris, Charles Pougens, An VI (1798), t. III, p. 24.

45. EM II, f. $17 \mathrm{r}^{\circ}$. 
tion - aux marges d'un monde cherchant à panser ses plaies par la promotion de l'esprit conquérant ${ }^{46}$. Un fragment des Pensées de Necker publiées par les soins de sa fille après son décès dépeint très exactement l'esprit de cette époque:

Les vieillards mènent une vie pénible, lorsqu'ils sont encore en état de tout apprécier, de tout sentir. La riante perspective de l'avenir ne leur appartient plus, et quand ils veulent parler du passé, on ne les écoute guères; chacun court vers les combats du monde, vers les champs de bataille d'où ils reviennent, c'est beaucoup quand on les salue en passant ${ }^{47}$.

Les deux versions de l'Entretien de Necker offrent ainsi un contrepoint troublant aux nombreuses études faisant des $\mathrm{XVIII}^{\mathrm{e}}$ et $\mathrm{XIX}^{\mathrm{e}}$ siècles l'époque d'une reconnaissance et d'une réhabilitation de la vieillesse ${ }^{48}$. Selon plusieurs chercheurs, la littérature ferait alors place à la figure du vieillard vénérable, pilier de sa famille, source de sagesse. Les fêtes révolutionnaires le célèbreraient, enfin de nouvelles mesures sociales et médicales viseraient à soulager ses maux et à augmenter son confort. La figure du vieillard apparaît alors encensée en plusieurs lieux. Si c'est bien en ces termes que Madame de Staël louera son père jusqu'à la fin de sa vie, on ne trouve rien de tel sous la plume de Necker, qui non seulement porte un regard extrêmement dépréciateur sur sa propre personne, mais redoute (à tort) le regard de sa fille («[...] en me supposant plus jeune, ce serait moi autant et plus quelle qui voudrais ne pas la quitter. Mais decrepit et connaissant l'impression que ma fille reçoit par les yeux... ${ }^{49} »$. L'autoportrait que brosse Necker pourrait être représentatif de cet écart signalé par des chercheurs tels que Jean-

46. Voir Michael J. Hughes, Forging Napoleon's Grande Armée. Motivation, Military Culture, and Masculinity in the French Army, 1800-1808, New York/London, New York University Press, 2012; Christopher E. Forth et Bertrand Taithe (édit.), French Masculinities. History, Culture and Politics, Basingstoke/New York, Palgrave Macmillan, 2007.

47. Jacques Necker, «21. Les vieillards», Manuscrits de M. ${ }^{r}$ Necker [...], op. cit., p. 49.

48. Voir par exemple David G. Troyansky, Miroirs de la vieillesse en France au siècle des Lumières, Paris, Eshel, 1992; Jean-Pierre Gutton, Naissance du vieillard. Essai sur l'histoire des rapports entre les vieillards et la société en France, Paris, Aubier, 1988; Simone de Beauvoir, La vieillesse, Paris, Gallimard, 1970.

49. EM II, f. $7 \mathrm{v}^{\circ}$. 
Pierre Bois ${ }^{50}$ et Philippe Ariès ${ }^{51}$, écart entre les représentations idéales de la vieillesse et la réalité historique, très variable selon les milieux et les circonstances individuelles. Le témoignage de Necker est à ce titre exemplaire. Selon l'auteur, le vieil homme cherchant à renouer avec une vie mondaine et publique quittée de longue date risque non seulement le ridicule, mais travaille à sa propre mort symbolique, et cela avec d'autant plus de risque s'il s'agit d'une personnalité publique: "C'est une grande resolution en general et pour soi et au jugement des autres que de se deplacer aussi completement après dix ou douze ans d'une même station et lorsqu'on est arrivé a l'epoque de la viellesse. Cette resolution est plus grande encore lorsqu'on s'est mis beaucoup en vue, et qu'on est presque devenu une sorte de personage histo-

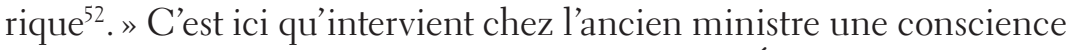
aiguë du statut rattaché à son passé d'homme d'État, par conséquent la nécessité absolue où il se trouve de préserver la considération acquise depuis sa retraite en Suisse, et qui seule a pu le dédommager d'une frange de l'opinion publique largement défavorable à ses initiatives politiques et littéraires. Necker sait qu'il n'aura pas sitôt mis le pied en France que les journalistes, tous ses anciens ennemis (parmi lesquels les Jacobins), enfin les membres du gouvernement en place ne manqueront pas une occasion de questionner les motifs de son retour et de l'attaquer:

Je ne dois pas me dissimuler que très injustement selon moi je me suis fait beaucoup d'ennemis et par l'effet de mes deux Administrations, et par les ouvrages libres ou austeres que j'ai publiés. Aussi tot que je quitterai ma retraite, aussi tot qu'on me verra reparaitre sur la scêne d'aucune maniere on repetera ce dicton repandu a dessein dans la classe des Emigrés et des Aristocrates «C'est lui qui est la premiere cause de la revolution $»^{53}$.

Revenir en France interdirait par ailleurs toute critique libre du gouvernement, que ce soit sous la forme écrite ou orale. À dire vrai, Necker éprouve une véritable « repugnance » à s'établir de nouveau «au milieu

50. Jean-Pierre Bois, Les vieux, de Montaigne aux premières retraites, Paris, Fayard, 1989.

51. Philippe Ariès, «Une histoire de la vieillesse? », Communications, 37, 1, 1983, p. $47-54$.

52. EM II, f. $8 \mathrm{r}^{\circ}$.

53. Id. 
d'un Pays où a peri d'une mort tragique et par des mains criminelles le Prince dont [il était] le Ministre». Lauteur redoute non seulement les souvenirs rattachés à cette tragédie, mais aussi le «blâme universel $^{54}$ » d'une majorité qui ne comprendrait sans doute pas son choix de revenir dans un pays à jamais marqué par le régicide. Le brouillon comme la version finale présentent encore un passage dans lequel Necker hallucine les conséquences funestes auxquelles une contrerévolution l'exposerait avec sa famille, loin du refuge coppétien :

Il faut puisque je compte tout et en bien et en mal, supposer le cas d'une revolution inattendue dans le Gouvernement, car si l'autorité passait a l'un ou a l'autre parti et quelle y passa a l'aide de moyens violens, j'aurais de justes sujets d'inquietude, surtout n'etant plus dans l'age où l'on peut s'echapper, et comme ma fille viendrait rester près de moi, la pensée que je l'empecherais d'etre libre dans ses mouvemens et de chercher une sureté qui lui serait a elle même necessaire me jetterait dans le desespoir ${ }^{55}$.

Tout autant que les craintes associées aux risques d'un renversement du gouvernement et à la perte de fortune qui pourrait en résulter, c'est la perspective de s'éloigner du tombeau de son épouse qui convainc Necker de ne pas quitter Coppet ${ }^{56}$. La seconde version développe considérablement cet argument et examine diverses possibilités, notamment celle de déplacer le tombeau en France, ou encore de le faire veiller en son absence et de prendre des mesures afin que sa propre dépouille soit jointe à celle de sa femme après son décès. L'auteur renonce à toutes ces options difficilement praticables en se remémorant les écrits de Madame Necker, sans équivoque sur son désir de n’être jamais séparée de son époux ${ }^{57}$.

54. Ibid., f. $10 \mathrm{v}^{\circ}-11 \mathrm{r}^{\circ}$.

55. Ibid., f. $10 \mathrm{r}^{\circ}$.

56. Afin de respecter les vœux de son épouse, qui lui avait fourni des instructions très spécifiques à cet égard, Necker a fait ériger un tombeau non loin du château (voir Gabriel-Paul-Othenin d'Haussonville, «Les dernières années de Madame Necker », Le salon de Madame Necker, Paris, Calmann Lévy, 1882, t. II, p. 287-303).

57. EM II, f. $12 v^{\circ}$, l'auteur souligne: «fCe que je vois retracé en plusieurs endroits dans les ecrits de mon amie ce sont ces paroles. Tu ne voudras pas que nous soyons separés. Rien n'a eté plus formel que ce vœu de sa part, mais elle supposait [a?] aussi que je pourrais la transporter dans le lieu où je prefererais de vivre. C'est dans son ouvrage sur le divorce ecrit a la verité lorsque les troubles de France semblaient nous fixer en Suisse, quelle se felicitait d'avoir son tombeau placé entre le dernier azile de 
Les manuscrits de l'Entretien confirment, s'il en était besoin, la nature inquiète de l'ancien homme d'État, prompt à se torturer, comme le montrait d'ailleurs, dès l'été 1785 , le court journal intime rédigé par sa fille, alors âgée de 19 ans. Dans ces pages, Germaine Necker insistait sur les remords affectant son père, en retraite à SaintOuen et Marolles, souffrant d'une première disgrâce auprès du roi, tenant sur sa vie publique un discours dépréciateur, mais cachant très mal son regret de ne plus y contribuer ${ }^{58}$. La jeune femme fantasmait alors le retour triomphal de son père au pouvoir, ne se doutant pas qu'elle en serait le premier témoin lorsque, en 1788, Louis XVI rappellerait son ancien ministre ${ }^{59}$. Les deux versions de l'Entretien, qui surviennent après la tempête révolutionnaire, sont d'un autre esprit. Écrites une quinzaine d'années après le Journal de jeunesse de Madame de Staël, elles montrent que Necker ne se fait plus aucune illusion sur sa capacité à réintégrer une vie politique ou une carrière dans les affaires. À plus d'une reprise, il évoque sa propre finitude:

Je tiens encore a ma retraite par le sentiment intime que je m'y suis amelioré. J'y ai fait des reflexions sur le monde, sur moi même sur les autres, sur les grands rapports de l'homme avec une cause Suprême, et ces reflexions ont perfectionné mon caractere, ont epuré mon ambition. Quelle transition de tout cela a Paris, a Paris encore tel qu'il est. Quelle transition vers la fin de sa vie et lorsqu'on a le plus besoin des sentimens qui doivent servir a nous consoler de notre decadence et a nous preparer pour la mort. - Oui dans ma retraite j’ai posé des pierres d'attente pour la route qu'il me reste a parcourir. Que deviendra tout cela si je retourne vers le monde, si je vais me placer au milieu de son tourbillon ${ }^{60}$.

La perspective d'un déplacement en France lui apparaît alors inconciliable avec toutes les facettes de cette préparation à la mort qui l'occupe

son pere et le dernier azile de sa mere. Elle n'avait pas non plus la prevoyance distincte des liens que formeraient autour de mon cœur ma fille et ses enfans.»

58. Voir Germaine Necker, «Mon journal », Cahiers staëliens, 28, 1980, «Le 16 [16 août 1785] », p. 76-77: «Faut-il me l'avouer à moi-même? Oui, je le crains, mon père n'aime pas tout ce qui lui rappelle une place qu'il regrette encore et comment ne pas la regretter avec une certitude aussi grande de ses talents. [...] cette place est entre nous comme une maîtresse infidèle: nous n'en disons que du mal, mais, si elle revenait, le langage changerait. »

59. Necker connaîtra trois ministères : 22 octobre 1776-19 mai 1781, 25 août 178811 juillet 1789 et 16 juillet 1789-3 septembre 1790 .

6o. EM II, f. $11 v^{\circ}-12 r^{\circ}$. 
en ce début de siècle, qu'il s'agisse de cultiver la paix de son cœur, de maintenir en ordre sa fortune en vue de la succession familiale, de soigner sa postérité d'homme d'État, de préserver une considération indispensable au bonheur de sa descendance ou encore d'honorer la mémoire de son épouse en ne s'éloignant pas de son tombeau. La rédaction à la même époque du Cours de morale religieuse (1800) vient cristalliser le désir de transmission, la volonté de partager son expérience de vie. Peu avant la fin du second Entretien, Necker affirme ne rien vouloir conclure puisqu'il a «sept ou huit mois devant [lui ${ }^{61}$ » avant de prendre sa décision. En dépit de ce commentaire, il ne fait aucun doute que l'auteur penche en faveur de la retraite et a sans doute déjà renoncé à la France. La suite de l'histoire le prouvera: Necker ne quittera plus jamais la Suisse, et c'est depuis son exil en Allemagne que Madame de Staël apprendra avec émois la mort de son père, survenue à Genève le 9 avril 1804 .

En définitive, les deux états de l'Entretien avec moi-même constituent des documents rares et d'une très grande valeur pour faire connaître Jacques Necker sous un jour privé. Ils se distinguent nettement de l'abondante production littéraire à laquelle ce dernier a habitué ses lecteurs ${ }^{62}$. S'il est vrai que Germaine et Auguste de Staël se sont employés à faire connaître la nature sensible de leur père et grand-père en éditant et en préfaçant ses œuvres de même qu'une part inédite de ses manuscrits, il reste que ce type d'écrits, de la part de Necker lui-même, est rarissime au sein des archives qui nous ont été léguées. On s'étonne qu'il n'ait pas davantage suscité l'attention des chercheurs, mais cela n'est sans doute pas étranger au caractère confidentiel du texte de même qu'à la tentation de maintenir intacte une image dont Necker était lui-même très soucieux. Ouvrir les pages de ces courts Entretiens, c'est prendre acte d'une fragilité, d'une vulnérabilité autrement cachées et qui, tôt ou tard, seront aussi les nôtres. C'est également rappeler la fonction capitale attribuée à l'écriture au sein de la famille Necker comme de la foi protestante, pratique indispensable à la connaissance de soi, à la lecture du cœur.

61. Ibid., f. $14 \mathrm{v}^{\circ}$.

62. Entre autres ouvrages, on retiendra le Compte rendu au roi (1781), De l'administration des finances de la France (1784), De l'importance des opinions religieuses (1788) et De la Révolution française (1796). 